\title{
BOTTOM OF THE $L^{2}$ SPECTRUM OF THE LAPLACIAN ON LOCALLY SYMMETRIC SPACES
}

\author{
JEAN-PHILIPPE ANKER AND HONG-WEI ZHANG
}

In memory of Michel Marias (1953-2020), who introduced us to locally symmetric spaces

\begin{abstract}
We estimate the bottom of the $L^{2}$ spectrum of the Laplacian on locally symmetric spaces in terms of the critical exponents of appropriate Poincare series. Our main result is the higher rank analog of a characterization due to Elstrodt, Patterson, Sullivan and Corlette in rank one. It improves upon previous results obtained by Leuzinger and Weber in higher rank.
\end{abstract}

\section{INTRODUCTION}

We adopt the standard notation and refer to [Hel00] for more details. Let $G$ be a semi-simple Lie group, connected, noncompact, with finite center, and $K$ be a maximal compact subgroup of $G$. The homogeneous space $X=G / K$ is a Riemannian symmetric space of noncompact type. Let $\mathfrak{g}=\mathfrak{k} \oplus \mathfrak{p}$ be the Cartan decomposition of the Lie algebra of $G$. The Killing form of $\mathfrak{g}$ induces a $K$-invariant inner product $\langle.,$.$\rangle on \mathfrak{p}$, hence a $G$-invariant Riemannian metric on $G / K$. Fix a maximal abelian subspace $\mathfrak{a}$ in $\mathfrak{p}$. We identify $\mathfrak{a}$ with its dual $\mathfrak{a}^{*}$ by means of the inner product inherited from $\mathfrak{p}$. Let $\Gamma$ be a discrete torsion-free subgroup of $G$ that acts freely and properly discontinuously on $X$. Then $Y=\Gamma \backslash X$ is a locally symmetric space, whose Riemannian structure is inherited from $X$. We denote by $d(.,$.$) the joint Riemannian distance$ on $X$ and $Y$, by $n$ their joint dimension, and by $\ell$ their joint rank, which is the dimension of $\mathfrak{a}$.

Let $\Sigma \subset \mathfrak{a}$ be the root system of $(\mathfrak{g}, \mathfrak{a})$ and let $W$ be the associated Weyl group. Choose a positive Weyl chamber $\mathfrak{a}^{+} \subset \mathfrak{a}$ and let $\Sigma^{+} \subset \Sigma$ be the corresponding subsystem of positive roots. Denote by $\rho=\frac{1}{2} \sum_{\alpha \in \Sigma^{+}} m_{\alpha} \alpha$ the half sum of positive roots counted with their multiplicities. Occasionally we shall need the reduced root system $\Sigma_{\text {red }}=\left\{\alpha \in \Sigma \mid \frac{\alpha}{2} \notin \Sigma\right\}$.

Consider the classical Poincaré series

$$
P_{s}(x K, y K)=\sum_{\gamma \in \Gamma} e^{-s d(x K, \gamma y K)} \quad \forall s>0, \forall x, y \in G
$$

and denote by $\delta(\Gamma)=\inf \left\{s>0 \mid P_{s}(x K, y K)<+\infty\right\}$ its critical exponent, which is independent of $x K$ and $y K$. Recall that $\delta(\Gamma) \in[0,2\|\rho\|]$ may be also defined by

$$
\delta(\Gamma)=\limsup _{R \rightarrow+\infty} \frac{\log N_{R}(x K, y K)}{R} \quad \forall x, y \in G,
$$

where $N_{R}(x K, y K)=|\{\gamma \in \Gamma \mid d(x K, \gamma y K) \leq R\}|$ denotes the orbital counting function. Finally, let $\Delta_{Y}$ be the Laplace-Beltrami operator on $Y$ and let $\lambda_{0}(Y)$ be the bottom of the $L^{2}$ spectrum of $-\Delta_{Y}$. The following celebrated result, due to Elstrodt ([Els73a], [Els73b], [Els74]), Patterson [Pat76], Sullivan [Sul87] and Corlette [Cor90], expresses $\lambda_{0}(Y)$ in terms of $\rho$ and $\delta(\Gamma)$ in rank one.

Theorem 1.1. In the rank one case $(\ell=1)$, we have

$$
\lambda_{0}(Y)= \begin{cases}\|\rho\|^{2} & \text { if } 0 \leq \delta(\Gamma) \leq\|\rho\|, \\ \|\rho\|^{2}-(\delta(\Gamma)-\|\rho\|)^{2} & \text { if }\|\rho\| \leq \delta(\Gamma) \leq 2\|\rho\| .\end{cases}
$$

2020 Mathematics Subject Classification. 22E40, 11N45, 35K08, 47A10, 58J50.

Key words and phrases. Locally symmetric space, $L^{2}$ spectrum, Poincaré series , critical exponent, Green function, heat kernel. 
This result was extended in higher rank as follows by Leuzinger [Leu04] and Weber [Web08]. Let $\rho_{\min }=\min _{H \in \overline{\mathfrak{a}}^{+},\|H\|=1}\langle\rho, H\rangle \in(0,\|\rho\|]$. Notice that $\rho_{\text {min }}=\|\rho\|$ in rank one and thus the following theorem reduces to Theorem 1.1.

Theorem 1.2. In the general case $(\ell \geq 1)$, the following estimates hold:

- Upper bound:

$$
\lambda_{0}(Y) \leq \begin{cases}\|\rho\|^{2} & \text { if } 0 \leq \delta(\Gamma) \leq\|\rho\|, \\ \|\rho\|^{2}-(\delta(\Gamma)-\|\rho\|)^{2} & \text { if }\|\rho\| \leq \delta(\Gamma) \leq 2\|\rho\| .\end{cases}
$$

- Lower bound:

$$
\lambda_{0}(Y) \geq \begin{cases}\|\rho\|^{2} & \text { if } 0 \leq \delta(\Gamma) \leq \rho_{\min }, \\ \max \left\{0,\|\rho\|^{2}-\left(\delta(\Gamma)-\rho_{\min }\right)^{2}\right\} & \text { if } \rho_{\min } \leq \delta(\Gamma) \leq 2\|\rho\| .\end{cases}
$$

In other terms,

$$
\begin{cases}\lambda_{0}(Y)=\|\rho\|^{2} & \text { if } \delta(\Gamma) \in\left[0, \rho_{\min }\right], \\ \lambda_{0}(Y) \in\left[\|\rho\|^{2}-\left(\delta(\Gamma)-\rho_{\min }\right)^{2},\|\rho\|^{2}\right] & \text { if } \delta(\Gamma) \in\left[\rho_{\min },\|\rho\|\right], \\ \lambda_{0}(Y) \in\left[\|\rho\|^{2}-\left(\delta(\Gamma)-\rho_{\min }\right)^{2},\|\rho\|^{2}-(\delta(\Gamma)-\|\rho\|)^{2}\right] & \text { if } \delta(\Gamma) \in\left[\|\rho\|,\|\rho\|+\rho_{\min }\right], \\ \lambda_{0}(Y) \in\left[0,\|\rho\|^{2}-(\delta(\Gamma)-\|\rho\|)^{2}\right] & \text { if } \delta(\Gamma) \in\left[\|\rho\|+\rho_{\min }, 2\|\rho\|\right] .\end{cases}
$$

In this paper, we first improve the lower bound of $\lambda_{0}(Y)$ in Theorem 1.2 by a slight modification of the classical Poincaré series (1.1). Let $\delta^{\prime}(\Gamma)$ denote the critical exponent of the modified Poincare series (2.7) associated to the polyhedral distance (2.1).

Theorem 1.3. The following lower bound holds for the bottom $\lambda_{0}(Y)$ of the $L^{2}$ spectrum of $-\Delta$ on $Y=\Gamma \backslash G / K$ :

$$
\lambda_{0}(Y) \geq \begin{cases}\|\rho\|^{2} & \text { if } 0 \leq \delta^{\prime}(\Gamma) \leq\|\rho\|, \\ \|\rho\|^{2}-\left(\delta^{\prime}(\Gamma)-\|\rho\|\right)^{2} & \text { if }\|\rho\| \leq \delta^{\prime}(\Gamma) \leq 2\|\rho\| .\end{cases}
$$

We obtain next a plain analog of Theorem 1.1 by considering a more involved family of Poincaré series. We denote by $\delta^{\prime \prime}(\Gamma)$ the critical exponent of $P_{s}^{\prime \prime}(x K, y K)$, see (3.3).

Theorem 1.4. The following characterization holds for the bottom $\lambda_{0}(Y)$ of the $L^{2}$ spectrum of $-\Delta$ on $Y=\Gamma \backslash G / K$ :

$$
\lambda_{0}(Y)= \begin{cases}\|\rho\|^{2} & \text { if } 0 \leq \delta^{\prime \prime}(\Gamma) \leq\|\rho\|, \\ \|\rho\|^{2}-\left(\delta^{\prime \prime}(\Gamma)-\|\rho\|\right)^{2} & \text { if }\|\rho\| \leq \delta^{\prime \prime}(\Gamma) \leq 2\|\rho\| .\end{cases}
$$

Remark 1.5. If $\Gamma$ is a lattice, i.e., $Y=\Gamma \backslash G / K$ has finite volume, then $\lambda_{0}(Y)=0$ and $\delta^{\prime \prime}(\Gamma)=2\|\rho\|$, hence $\delta^{\prime}(\Gamma)=2\|\rho\|$. Furthermore $\delta(\Gamma)=2\|\rho\|$ [Alb99, Theorem 7.4]. As pointed out by Corlette [Cor90] in rank one and by Leuzinger [Leu03] in higher rank, if G has Kazhdan's property $(T)$, then the following conditions are actually equivalent:
(a) $\Gamma$ is a lattice,
(b) $\lambda_{0}(Y)=0$,
(c) $\delta(\Gamma)=2\|\rho\|$,
(d) $\delta^{\prime \prime}(\Gamma)=2\|\rho\|$.

Remark 1.6. As for the Green function, the heat kernel

$$
h_{t}^{Y}(\Gamma x K, \Gamma y K)=\sum_{\gamma \in \Gamma} h_{t}\left(K y^{-1} \gamma^{-1} x K\right)
$$

on a locally symmetric space $Y=\Gamma \backslash G / K$ can be expressed and estimated by using the heat kernel $h_{t}$ on the symmetric space $X=G / K$, whose behavior is well understood [AnJi99; AnOs03]. By adapting straightforwardly the methods carried out in [DaMa88; Web08], and by applying Theorem 1.4 instead of Theorem 1.1 and Theorem 1.2, we refine the Gaussian bounds of $h_{t}^{Y}$ 
and get rid in particular of $\rho_{\min }$. The following estimates hold for all $t>0$ and all $x, y \in G$ :

(i) Assume that $\delta^{\prime \prime}(\Gamma)<\|\rho\|$ and let $\delta^{\prime \prime}(\Gamma)<s<\|\rho\|$. Then

$$
h_{t}^{Y}(\Gamma x K, \Gamma y K) \lesssim t^{-\frac{n}{2}}(1+t)^{\frac{n-D}{2}} e^{-\|\rho\|^{2} t} e^{-\frac{d(\Gamma x K, \Gamma y K)^{2}}{4 t}} P_{s}^{\prime \prime}(x K, y K) .
$$

(ii) Assume that $\|\rho\| \leq \delta^{\prime \prime}(\Gamma)<2\|\rho\|$ and let $\delta^{\prime \prime}(\Gamma)-\|\rho\|<s_{1}<s_{2}<\|\rho\|$. Then

$$
h_{t}^{Y}(\Gamma x K, \Gamma y K) \lesssim t^{-\frac{n}{2}} e^{-\left(\|\rho\|^{2}-s_{2}^{2}\right) t} P_{\|\rho\|+s_{1}}^{\prime \prime}(x K, y K) .
$$

(iii) Assume that $\delta^{\prime \prime}(\Gamma)<2\|\rho\|$. Let $s>\delta^{\prime \prime}(\Gamma)$ and $\varepsilon>0$. Then

$$
h_{t}^{Y}(\Gamma x K, \Gamma y K) \lesssim t^{-\frac{n}{2}} e^{-\left(\|\rho\|^{2}-\left(\delta^{\prime \prime}(\Gamma)-\|\rho\|\right)^{2}-2 \varepsilon\right) t} e^{-\frac{d(\Gamma x K, \Gamma y K)^{2}}{4(1+\varepsilon) t}} P_{s}^{\prime \prime}(x K, x K)^{\frac{1}{2}} P_{s}^{\prime \prime}(y K, y K)^{\frac{1}{2}} .
$$

\section{First improvement}

In this section, we replace the Riemannian distance $d$ on $X$ by a polyhedral distance

$$
d^{\prime}(x K, y K)=\left\langle\frac{\rho}{\|\rho\|},\left(y^{-1} x\right)^{+}\right\rangle \quad \forall x, y \in G,
$$

where $\left(y^{-1} x\right)^{+}$denotes the $\overline{\mathfrak{a}^{+}}$-component of $y^{-1} x$ in the Cartan decomposition $G=K\left(\exp \overline{\mathfrak{a}^{+}}\right) K$. The corresponding balls, which reflect the volume growth of $X$ at infinity, played an important role in [Ank90] and [Ank91]. More general polyhedral sets were considered in [Ank92] and [AAS10].

Proposition 2.1. $d^{\prime}$ is a $G$-invariant distance on $X$.

Proof. Notice first that (2.1) descends from $G \times G$ to $X \times X$, as the map $z \mapsto z^{+}$is $K$-bi-invariant on $G$. The $G$-invariance of $d^{\prime}$ is straightforward from the definition (2.1). The symmetry

$$
d^{\prime}(x K, y K)=d^{\prime}(y K, x K)
$$

follows from

$$
\left(y^{-1}\right)^{+}=-w_{0} \cdot y^{+} \text {and } \quad-w_{0} \cdot \rho=\rho,
$$

where $w_{0}$ denotes the longest element in the Weyl group. Let us check the triangular inequality

$$
d^{\prime}(x K, y K) \leq d^{\prime}(x K, z K)+d^{\prime}(z K, y K) .
$$

By $G$-invariance, we may reduce to the case where $z K=e K$. According to Lemma 2.2 below,

$$
x^{+}+\left(y^{-1}\right)^{+}-\left(y^{-1} x\right)^{+}
$$

belongs to the cone generated by the positive roots.

Lemma 2.2. For every $x, y \in G$, we have the following inclusion

$$
\operatorname{co}\left[W \cdot(x y)^{+}\right] \subset \operatorname{co}\left[W \cdot\left(x^{+}+y^{+}\right)\right]
$$

between convex hulls.

Proof. The inclusion (2.3) amounts to the fact that

$$
x^{+}+y^{+}-(x y)^{+}
$$

belongs to the cone generated by the positive roots or, equivalently, to the inequality

$$
\left\langle\lambda,(x y)^{+}\right\rangle \leq\left\langle\lambda, x^{+}\right\rangle+\left\langle\lambda, y^{+}\right\rangle \quad \forall \lambda \in \overline{\mathfrak{a}^{+}} .
$$

It is enough to prove (2.4) for all highest weights $\lambda$ of irreducible finite-dimensional complex representations $\pi: G \longrightarrow \mathrm{GL}(V)$ with $K$-fixed vectors. According to Weyl's unitary trick (see for instance [Kna02, Proposition 7.15]), there exists an inner product on $V$ such that

$$
\begin{cases}\pi(k) \text { is unitary } & \forall k \in K, \\ \pi(a) \text { is self-adjoint } & \forall a \in \exp \mathfrak{a} .\end{cases}
$$


As $\lambda$ is the highest weight of $\pi$, then

$$
e^{\left\langle\lambda,(x y)^{+}\right\rangle}=\|\pi(x y)\| \leq\|\pi(x)\|\|\pi(y)\|=e^{\left\langle\lambda, x^{+}\right\rangle} e^{\left\langle\lambda, y^{+}\right\rangle}=e^{\left\langle\lambda, x^{+}+y^{+}\right\rangle} .
$$

Remark 2.3. The distance $d^{\prime}$ is comparable to the Riemannian distance $d$. Specifically,

$$
\frac{\rho_{\min }}{\|\rho\|} d(x K, y K) \leq d^{\prime}(x K, y K) \leq d(x K, y K) \quad \forall x, y \in G \text {. }
$$

This follows indeed from

$$
\frac{\rho_{\min }}{\|\rho\|}\|H\| \leq\left\langle\frac{\rho}{\|\rho\|}, H\right\rangle \leq\|H\| \quad \forall H \in \overline{\mathfrak{a}^{+}} .
$$

The volume of balls

$$
B_{r}^{\prime}(x K)=\left\{y K \in X \mid d^{\prime}(y K, x K) \leq r\right\}
$$

was determined in [Ank90, Lemma 6]. For the reader's convenience, we recall the statement and its proof.

Lemma 2.4. For every $x \in G$ and $r>0$, we have ${ }^{1}$

$$
\left|B_{r}^{\prime}(x K)\right| \asymp \begin{cases}r^{n} & \text { if } 0<r<1, \\ r^{\ell-1} e^{2\|\rho\| r} & \text { if } r \geq 1 .\end{cases}
$$

Remark 2.5. Notice the different large scale behavior, in comparison with the classical ball volume

$$
\left|B_{r}(x K)\right| \asymp \begin{cases}r^{n} & \text { if } 0<r<1, \\ r^{\frac{\ell-1}{2}} e^{2\|\rho\| r} & \text { if } r \geq 1,\end{cases}
$$

see for instance [Str81] or [Kni9'7].

Proof. By translation invariance, we may assume that $x=e$. Recall the integration formula

$$
\int_{X} d x f(x)=\text { const. } \int_{K} d k \int_{\mathfrak{a}^{+}} d H \omega(H) f(k(\exp H) K),
$$

in the Cartan decomposition, with density

$$
\omega(H)=\prod_{\alpha \in \Sigma^{+}}(\sinh \langle\alpha, H\rangle)^{m_{\alpha}} \asymp \prod_{\alpha \in \Sigma^{+}}\left(\frac{\langle\alpha, H\rangle}{1+\langle\alpha, H\rangle}\right)^{m_{\alpha}} e^{2\langle\rho, H\rangle},
$$

since $\sinh t \sim \frac{t}{1+t} e^{t}$ for all $t \geq 0$. Thus

$$
\left|B_{r}^{\prime}(e K)\right|=\text { const. } \int_{\left\{H \in \mathfrak{a}^{+} \mid\langle\rho, H\rangle \leq\|\rho\| r\right\}} d H \omega(H) \asymp \int_{\left\{H \in \mathfrak{a}^{+} \mid\|H\| \leq r\right\}} d H \prod_{\alpha \in \Sigma^{+}}\langle\alpha, H\rangle^{m_{\alpha}} \asymp r^{n}
$$

if $r$ is small. Let us turn to $r$ large. On the one hand, we estimate from above

$$
\left|B_{r}^{\prime}(e K)\right| \lesssim \int_{\left\{H \in \mathfrak{a}^{+} \mid\langle\rho, H\rangle \leq\|\rho\| r\right\}} d H e^{2\langle\rho, H\rangle} \asymp \int_{0}^{2\|\rho\| r} d s s^{\ell-1} e^{s} \asymp r^{\ell-1} e^{2\|\rho\| r} .
$$

On the other hand, let $H_{0} \in \mathfrak{a}^{+}$. As

$$
\omega(H) \asymp e^{2\langle\rho, H\rangle} \quad \forall H \in H_{0}+\overline{\mathfrak{a}^{+}},
$$

we estimate from below

$$
\left|B_{r}^{\prime}(e K)\right| \gtrsim \int_{\left\{H \in H_{0}+\mathfrak{a}^{+} \mid\langle\rho, H\rangle \leq\|\rho\| r\right\}} d H e^{2\langle\rho, H\rangle} \gtrsim \int_{C_{0}}^{2\|\rho\| r} d s s^{\ell-1} e^{s} \asymp r^{\ell-1} e^{2\|\rho\| r},
$$

where $C_{0}>0$ is a constant depending on $H_{0}$.

\footnotetext{
${ }^{1}$ The symbol $f \asymp g$ between two non-negative expressions means that there exist constants $0<A \leq B<+\infty$ such that $A g \leq f \leq B g$.
} 
Consider now the modified Poincaré series

$$
P_{s}^{\prime}(x K, y K)=\sum_{\gamma \in \Gamma} e^{-s d^{\prime}(x K, \gamma y K)} \quad \forall s>0, \forall x, y \in G
$$

associated with $d^{\prime}$, its critical exponent

$$
\delta^{\prime}(\Gamma)=\inf \left\{s>0 \mid P_{s}^{\prime}(x K, y K)<+\infty\right\}
$$

and the modified orbital counting function

$$
N_{R}^{\prime}(x K, y K)=\left|\left\{\gamma \in \Gamma \mid d^{\prime}(x K, \gamma y K) \leq R\right\}\right| \quad \forall R \geq 0, \forall x, y \in G .
$$

The following proposition shows that (2.7), (2.8) and (2.9) share the properties of their classical counterparts.

Proposition 2.6. The following assertions hold:

(i) $\delta^{\prime}(\Gamma)$ does not depend on the choice of $x$ and $y$.

(ii) $0 \leq \delta^{\prime}(\Gamma) \leq 2\|\rho\|$.

(iii) For every $x, y \in G$,

$$
\delta^{\prime}(\Gamma)=\limsup _{R \rightarrow+\infty} \frac{\log N_{R}^{\prime}(x K, y K)}{R} .
$$

Remark 2.7. It follows from (2.5) that

$$
P_{s}(x K, y K) \leq P_{s}^{\prime}(x K, y K) \leq P_{\frac{\rho_{\min }}{\|\rho\|} s}(x K, y K)
$$

and

$$
N_{R}(x K, y K) \leq N_{R}^{\prime}(x K, y K) \leq N_{\frac{\|\rho\|}{\rho_{\min }}}(x K, y K)
$$

Hence

$$
0 \leq \delta(\Gamma) \leq \delta^{\prime}(\Gamma) \leq \frac{\|\rho\|}{\rho_{\min }} \delta(\Gamma) .
$$

Proof. (i) follows from the triangular inequality. More precisely, let $x_{1}, y_{1}, x_{2}, y_{2} \in G$ and $s>0$. Then

$$
d^{\prime}\left(x_{2} K, \gamma y_{2} K\right) \leq d^{\prime}\left(x_{2} K, x_{1} K\right)+d^{\prime}\left(x_{1} K, \gamma y_{1} K\right)+\underbrace{d^{\prime}\left(\gamma y_{1} K, \gamma y_{2} K\right)}_{d^{\prime}\left(y_{1} K, y_{2} K\right)} \quad \forall \gamma \in \Gamma,
$$

hence

$$
\underbrace{\sum_{\gamma \in \Gamma} e^{-s d^{\prime}\left(x_{1} K, \gamma y_{1} K\right)}}_{P_{s}^{\prime}\left(x_{1} K, y_{1} K\right)} \leq e^{s\left\{d^{\prime}\left(x_{1} K, x_{2} K\right)+d^{\prime}\left(y_{1} K, y_{2} K\right)\right\}} \underbrace{\sum_{\gamma \in \Gamma} e^{-s d^{\prime}\left(x_{2} K, \gamma y_{2} K\right)}}_{P_{s}^{\prime}\left(x_{2} K, y_{2} K\right)} .
$$

(ii) According to (i), let us show, without loss of generality, that $P_{s}^{\prime}(e K, e K)<+\infty$ for every $s>2\|\rho\|$. According to Lemma 2.9 below, there exists $r>0$ such that the balls $B_{r}^{\prime}(\gamma K)$, with $\gamma \in \Gamma$, are pairwise disjoint in $G / K$. Let us apply the integration formula (2.6) to the function

$$
f_{s}(x K)=\sum_{\gamma \in \Gamma} e^{-s d^{\prime}(\gamma K, e K)} \mathbf{1}_{B_{r}^{\prime}(\gamma K)}(x K) .
$$

On the one hand, as

$$
\left|d^{\prime}(x K, e K)-d^{\prime}(\gamma K, e K)\right| \leq r \quad \forall x K \in B_{r}^{\prime}(\gamma K),
$$

we have

$$
\int_{X} d(x K) f_{s}(x K) \asymp \sum_{\gamma \in \Gamma} e^{-s d^{\prime}(\gamma K, e K)} \underbrace{\left|B_{r}^{\prime}(\gamma K)\right|}_{\left|B_{r}^{\prime}(e K)\right|} \asymp P_{s}^{\prime}(e K, e K) .
$$


On the other hand,

$$
\begin{aligned}
\int_{X} d(x K) f_{s}(x K) & \lesssim \int_{X} d(x K) e^{-s d^{\prime}(x K, e K)} \\
& \asymp \int_{\mathfrak{a}^{+}} d H \omega(H) e^{-s\left\langle\frac{\rho}{\|\rho\|}, H\right\rangle} \lesssim \int_{\mathfrak{a}^{+}} d H e^{-\left(\frac{s}{\|\rho\|}-2\right)\langle\rho, H\rangle}
\end{aligned}
$$

is finite if $s>2\|\rho\|$. Thus $P_{s}^{\prime}(e K, e K)<+\infty$ in that case, and consequently $\delta^{\prime}(\Gamma) \leq 2\|\rho\|$.

(iii) Denote the right hand side of $(2.10)$ by $L(x K, y K)$ and let us first show that $L(x K, y K)$ is finite. By applying Lemma 2.9 below to $y^{-1} \Gamma y$, we deduce that there exists $r>0$ such that the balls $B_{r}^{\prime}(\gamma y K)$, with $\gamma \in \Gamma$, are pairwise disjoint. Set

$$
\Gamma_{R}^{\prime}(x K, y K)=\left\{\gamma \in \Gamma \mid d^{\prime}(x K, \gamma y K) \leq R\right\} \quad \forall R \geq 0, \forall x, y \in G .
$$

Then the ball $B_{R+r}^{\prime}(x K)$ contains the disjoint balls $B_{r}^{\prime}(\gamma y K)$, with $\gamma \in \Gamma_{R}^{\prime}(x K, y K)$. By computing volumes, we estimate

$$
N_{R}^{\prime}(x K, y K)=\left|\Gamma_{R}^{\prime}(x K, y K)\right| \leq \frac{\left|B_{R+r}^{\prime}(x K)\right|}{\left|B_{r}^{\prime}(e K)\right|} \asymp(1+R)^{\ell-1} e^{2\|\rho\| R} .
$$

Hence $L(x K, y K) \leq 2\|\rho\|$. Let us next show that $L(x K, y K)$ is actually independent of $x, y \in G$. Given $x_{1}, y_{1}, x_{2}, y_{2} \in G$ and $R_{1}>0$, let

$$
R_{2}=R_{1}+d^{\prime}\left(x_{1} K, x_{2} K\right)+d^{\prime}\left(y_{1} K, y_{2} K\right) .
$$

Then the triangular inequality

$$
d^{\prime}\left(x_{2} K, \gamma y_{2} K\right) \leq d^{\prime}\left(x_{2} K, x_{1} K\right)+d^{\prime}\left(x_{1} K, \gamma y_{1} K\right)+\underbrace{d^{\prime}\left(\gamma y_{1} K, \gamma y_{2} K\right)}_{d^{\prime}\left(y_{1} K, y_{2} K\right)}
$$

implies successively

$$
\begin{aligned}
\Gamma_{R_{1}}^{\prime}\left(x_{1} K, y_{1} K\right) & \subset \Gamma_{R_{2}}^{\prime}\left(x_{2} K, y_{2} K\right), \\
N_{R_{1}}^{\prime}\left(x_{1} K, y_{1} K\right) & \leq N_{R_{2}}^{\prime}\left(x_{2} K, y_{2} K\right), \\
L\left(x_{1} K, y_{1} K\right) & \leq L\left(x_{2} K, y_{2} K\right) .
\end{aligned}
$$

Let us finally prove the equality between $\delta^{\prime}(\Gamma)$ and $L=L(e K, e K)$. For this purpose, observe that

$$
\begin{aligned}
P_{s}^{\prime} & =1+\sum_{R \in \mathbb{N}^{*}} \sum_{\gamma \in \Gamma_{R}^{\prime} \backslash \Gamma_{R-1}^{\prime}} e^{-s d^{\prime}(e K, \gamma K)} \\
& \asymp 1+\sum_{R \in \mathbb{N}^{*}}\left(N_{R}^{\prime}-N_{R-1}^{\prime}\right) e^{-s R} \\
& \asymp \sum_{R \in \mathbb{N}^{-s}} N_{R}^{\prime} e^{-s R},
\end{aligned}
$$

where we have written for simplicity

$$
P_{s}^{\prime}=P_{s}^{\prime}(e K, e K), \quad \Gamma_{R}^{\prime}=\Gamma_{R}^{\prime}(e K, e K) \quad \text { and } \quad N_{R}^{\prime}=N_{R}^{\prime}(e K, e K) .
$$

One the one hand, let $s>L$ and set $\varepsilon=\frac{s-L}{2}$. By definition of $L$,

$$
N_{R}^{\prime} \lesssim e^{(L+\varepsilon) R} \quad \forall R \geq 0 .
$$

Hence

$$
P_{s}^{\prime} \lesssim \sum_{R \in \mathbb{N}} e^{-\varepsilon R}<+\infty
$$

One the other hand, let $s<L$. By definition of $L$, there exists a sequence of integers $1<R_{1}<$ $R_{2}<\cdots \rightarrow+\infty$ such that

$$
N_{R_{j}}^{\prime} \geq e^{s R_{j}} \quad \forall j \in \mathbb{N}^{*} .
$$

Hence the series (2.11) diverges. 
Remark 2.8. Here is an example where $\delta<\delta^{\prime}$. Consider the product

$$
\Gamma \backslash G / K=\left(\Gamma_{1} \backslash G_{1} / K_{1}\right) \times\left(\Gamma_{2} \backslash G_{2} / K_{2}\right)
$$

of two locally symmetric spaces of rank one, with parameters $\rho_{1}, \delta_{1}$ and $\rho_{2}, \delta_{2}$. Then

$$
\delta \leq \sqrt{\delta_{1}^{2}+\delta_{2}^{2}}
$$

and

$$
\delta^{\prime} \geq \sqrt{\rho_{1}^{2}+\rho_{2}^{2}} \max \left\{\frac{\delta_{1}}{\rho_{1}}, \frac{\delta_{2}}{\rho_{2}}\right\} .
$$

Hence $\delta<\delta^{\prime}$ if $\frac{\delta_{1}}{\rho_{1}} \neq \frac{\delta_{2}}{\rho_{2}}$. Notice that there are plenty of such products, starting with the case where $\delta_{1}=2 \rho_{1}$ and $\delta_{2}=0$. Let us first prove (2.12) and begin with some notation. Write for simplicity

$$
N_{1, R}=\left(N_{1}\right)_{R}\left(e K_{1}, e K_{1}\right), N_{2, R}=\left(N_{2}\right)_{R}\left(e K_{2}, e K_{2}\right) \text { and } N_{R}=N_{R}(e K, e K),
$$

for every $R \geq 0$. Moreover, for every $D \subset \mathbb{R}_{+}^{2}$, denote by $N(D)$ the number of $\gamma=\left(\gamma_{1}, \gamma_{2}\right)$ in $\Gamma=\Gamma_{1} \times \Gamma_{2}$ such that $\left(d_{1}\left(\gamma_{1} K_{1}, e K_{1}\right), d_{2}\left(\gamma_{2} K_{2}, e K_{2}\right)\right)$ belongs to $D$. In $\mathbb{R}_{+}^{2}$ we consider the covering of $D_{R}=\left\{\left(R_{1}, R_{2}\right) \in \mathbb{R}_{+}^{2} \mid R_{1}^{2}+R_{2}^{2} \leq R^{2}\right\}$ by the two segments $[0, R] \times\{0\},\{0\} \times[0, R]$ and by the squares $Q_{j_{1}, j_{2}}=\left(j_{1}, j_{1}+1\right] \times\left(j_{2}, j_{2}+1\right]$, with $j_{1}^{2}+j_{2}^{2}<R^{2}$. Then

$$
N_{R}=N\left(D_{R}\right) \leq N_{1, R}+N_{2, R}+\sum_{j_{1}^{2}+j_{2}^{2}<R^{2}} N\left(Q_{j_{1}, j_{2}}\right)
$$

with

$$
N\left(Q_{j_{1}, j_{2}}\right)=\left(N_{1, j_{1}+1}-N_{1, j_{1}}\right)\left(N_{2, j_{2}+1}-N_{2, j_{2}}\right) \leq N_{1, j_{1}+1} N_{2, j_{2}+1} .
$$

Given $s_{1}>\delta_{1}$ and $s_{2}>\delta_{2}$, there exist $C \geq 1$ such that

$$
N_{1, R} \leq C e^{s_{1} R} \quad \text { and } \quad N_{2, R} \leq C e^{s_{2} R}
$$

for every $R \geq 0$. By combining (2.14), (2.15) and (2.16), we get

$$
N_{R} \leq C e^{s_{1} R}+C e^{s_{2} R}+C^{2} \sum_{j_{1}^{2}+j_{2}^{2}<R^{2}} e^{s_{1}\left(j_{1}+1\right)+s_{2}\left(j_{2}+1\right)} .
$$

Up to a multiplicative constant, the right hand side of (2.17) is bounded above by the integral

$$
\int_{\mathbb{R}_{+}^{2} \cap B(0, R+2)} d R_{1} d R_{2} e^{s_{1} R_{1}+s_{2} R_{2}}=\int_{0}^{R+2} d r r \int_{0}^{\frac{\pi}{2}} d \theta e^{r\left(s_{1} \cos \theta+s_{2} \sin \theta\right)} .
$$

As the function $\theta \longmapsto s_{1} \cos \theta+s_{2} \sin \theta$ reaches its maximum $\sqrt{s_{1}^{2}+s_{2}^{2}}$ at $\theta_{0}=\arctan \frac{s_{2}}{s_{1}}$, the latter integral is itself bounded above by

$$
\frac{\pi}{2} \int_{0}^{R+2} d r r e^{r \sqrt{s_{1}^{2}+s_{2}^{2}}} \leq \frac{\pi}{2} \frac{R+2}{\sqrt{s_{1}^{2}+s_{2}^{2}}} e^{(R+2) \sqrt{s_{1}^{2}+s_{2}^{2}}}
$$

In conclusion, we obtain

$$
\frac{\log N_{R}}{R} \leq \frac{2 \log C+\log \pi-\log 2-\frac{1}{2} \log \left(s_{1}^{2}+s_{2}^{2}\right)}{R}+\frac{\log (R+2)}{R}+\frac{R+2}{R} \sqrt{s_{1}^{2}+s_{2}^{2}}
$$

by combining (2.17), (2.18) and (2.19), hence $\delta \leq \sqrt{s_{1}^{2}+s_{2}^{2}}$ by letting $R \rightarrow+\infty$, and finally $\delta \leq \sqrt{\delta_{1}^{2}+\delta_{2}^{2}}$ by letting $s_{1} \searrow \delta_{1}$ and $s_{2} \searrow \delta_{2}$. Let us turn to the proof of (2.13). Set $\|\rho\|=$ $\sqrt{\rho_{1}^{2}+\rho_{2}^{2}}$ and assume that $\frac{\delta_{1}}{\rho_{1}} \geq \frac{\delta_{2}}{\rho_{2}}$. As

$$
d^{\prime}(\gamma K, e K)=\frac{\rho_{1}}{\|\rho\|} d_{1}\left(\gamma_{1} K_{1}, e K_{1}\right)+\frac{\rho_{2}}{\|\rho\|} d_{2}\left(\gamma_{2} K_{2}, e K_{2}\right),
$$

the set $\left\{\gamma \in \Gamma \mid d^{\prime}(\gamma K, e K) \leq R\right\}$ contains the product

$$
\left\{\gamma_{1} \in \Gamma_{1} \mid d_{1}\left(\gamma_{1} K_{1}, e K_{1}\right) \leq \frac{\|\rho\|}{\rho_{1}} R\right\} \times\{e\},
$$

for every $R \geq 0$. Hence $N_{R}^{\prime} \geq N_{1, \frac{\|\rho\|}{\rho_{1}} R}$, where $N_{R}^{\prime}=N_{R}^{\prime}(e K, e K)$, and conseqently $\delta^{\prime} \geq \frac{\|\rho\|}{\rho_{1}} \delta_{1}$. 
Lemma 2.9. ${ }^{2}$ There exists $r>0$ such that the balls $B_{r}^{\prime}(\gamma K)$, with $\gamma \in \Gamma$, are pairwise disjoint in $G / K$.

Proof. Let $r>0$. As $\Gamma$ is discrete in $G$, its intersection with the compact subset

$$
G_{r}^{\prime}=\left\{y \in G \mid d^{\prime}(y K, e K) \leq r\right\}=K\left(\exp \left\{H \in \overline{\mathfrak{a}^{+}} \mid\langle\rho, H\rangle \leq\|\rho\| r\right\}\right) K
$$

is finite. Moreover, as $\Gamma$ is torsion-free,

$$
\gamma^{+} \neq 0 \quad \forall \gamma \in \Gamma \backslash\{e\} .
$$

Hence there exists $r>0$ such that $\Gamma \cap G_{2 r}^{\prime}=\{e\}$, which implies that the sets $\gamma G_{r}^{\prime}$ are pairwise disjoint in $G$. In other words, the balls $B_{r}^{\prime}(\gamma K)$ are pairwise disjoint in $G / K$.

By using $\delta^{\prime}(\Gamma)$, we prove Theorem 1.3, which improves the lower bound in Theorem 1.2.

Proof of Theorem 1.3. Let us resume the approach in [Cor90, Section 4] and [Leu04, Section 3]. It consists in studying the convergence of the positive series

$$
g_{\zeta}^{\Gamma}(\Gamma x K, \Gamma y K)=\sum_{\gamma \in \Gamma} g_{\zeta}\left(K y^{-1} \gamma^{-1} x K\right),
$$

which expresses the kernel $g_{\zeta}^{\Gamma}$ of $\left(-\Delta-\|\rho\|^{2}+\zeta^{2}\right)^{-1}$ on the locally symmetric space $Y=\Gamma \backslash G / K$ in terms of the corresponding Green function $g_{\zeta}$ on the symmetric space $X=G / K$. Here $\zeta>0$ and $\Gamma x K \neq \Gamma y K$. Recall [AnJi99, Theorem 4.2.2] that

$$
g_{\zeta}(\exp H) \asymp\left\{\prod_{\alpha \in \Sigma_{\text {red }}^{+}}(1+\langle\alpha, H\rangle)\right\}\|H\|^{-\frac{\ell-1}{2}-\left|\Sigma_{\text {red }}^{+}\right|} e^{-\langle\rho, H\rangle-\zeta\|H\|}
$$

for $H \in \overline{\mathfrak{a}^{+}}$large, let say $\|H\| \geq \frac{1}{2}$, while

$$
g_{\zeta}(\exp H) \asymp \begin{cases}\|H\|^{-(n-2)} & \text { if } n>2 \\ \log \frac{1}{\|H\|} & \text { if } n=2\end{cases}
$$

for $H$ small, lets say $0<\|H\| \leq \frac{1}{2}$. Thus (2.20) converges if and only if

$$
\begin{aligned}
\sum_{\gamma \in \Gamma} & \left\{\prod_{\alpha \in \Sigma_{\text {red }}^{+}}\left(1+\left\langle\alpha,\left(y^{-1} \gamma^{-1} x\right)^{+}\right\rangle\right)\right\} \times \\
& \times d(x K, \gamma y K)^{-\frac{\ell-1}{2}-\left|\Sigma_{\text {red }}^{+}\right|} e^{-\|\rho\| d^{\prime}(x K, \gamma y K)-\zeta d(x K, \gamma y K)}
\end{aligned}
$$

converges. Let us compare the series (2.22) with the Poincaré series (1.1) and (2.7). On the one hand, as $\left\|\left(y^{-1} \gamma^{-1} x\right)^{+}\right\|=d(x K, \gamma y K),(2.22)$ is bounded from above by $P_{\|\rho\|+\zeta}^{\prime}(x K, y K)$. On the other hand, as

$$
d(x K, \gamma y K)^{-\frac{\ell-1}{2}-\left|\Sigma_{\text {red }}^{+}\right|} \gtrsim e^{-\varepsilon d(x K, \gamma y K)}
$$

for every $\varepsilon>0,(2.22)$ is bounded from below by $P_{\|\rho\|+\zeta+\varepsilon}(x K, y K)$. Hence (2.22) converges if $\|\rho\|+\zeta>\delta^{\prime}(\Gamma)$, i.e., $\zeta>\delta^{\prime}(\Gamma)-\|\rho\|$, while (2.22) diverges if $\zeta<\delta(\Gamma)-\|\rho\|$. We conclude by using the fact [Cor90, Section 4] that $\lambda_{0}(Y)$ is the supremum of $\|\rho\|^{2}-\zeta^{2}$ over all $\zeta>0$ such that (2.20) converges.

Next statement is obtained by combining this lower bound with the upper bound in Theorem 1.2 .

Corollary 2.10. The following estimates hold for $\lambda_{0}(Y)$ :

$$
\begin{cases}\lambda_{0}(Y)=\|\rho\|^{2} & \text { if } \delta^{\prime}(\Gamma) \leq\|\rho\|, \\ \|\rho\|^{2}-\left(\delta^{\prime}(\Gamma)-\|\rho\|\right)^{2} \leq \lambda_{0}(Y) \leq\|\rho\|^{2} & \text { if } \delta(\Gamma) \leq\|\rho\| \leq \delta^{\prime}(\Gamma), \\ \|\rho\|^{2}-\left(\delta^{\prime}(\Gamma)-\|\rho\|\right)^{2} \leq \lambda_{0}(Y) \leq\|\rho\|^{2}-(\delta(\Gamma)-\|\rho\|)^{2} & \text { if }\|\rho\| \leq \delta(\Gamma) .\end{cases}
$$

\footnotetext{
${ }^{2}$ As observed by the referee, Lemma 3 still holds without the torsion-free assumption, provided that $\gamma$ runs through $\Gamma \backslash(\Gamma \cap K)$.
} 


\section{SECOND IMPROVEMENT}

In this section, we obtain the actual higher rank analog of Theorem 1.1 by considering a further family of distances on $X$, which reflects the large scale behavior (2.21) of the Green function. Specifically, for every $s>0$ and $x, y \in G$, let

$$
\begin{aligned}
d_{s}^{\prime \prime}(x K, y K) & =\min \{s,\|\rho\|\} d^{\prime}(x K, y K)+\max \{s-\|\rho\|, 0\} d(x K, y K) \\
& = \begin{cases}s d^{\prime}(x K, y K) & \text { if } 0<s \leq\|\rho\|, \\
\|\rho\| d^{\prime}(x K, y K)+(s-\|\rho\|) d(x K, y K) & \text { if } s \geq\|\rho\| .\end{cases}
\end{aligned}
$$

Then (3.1) defines a $G$-invariant distance on $X$ such that

$$
s d^{\prime}(x K, y K) \leq d_{s}^{\prime \prime}(x K, y K) \leq s d(x K, y K) \quad \forall s>0, \forall x, y \in G .
$$

Consider the associated Poincaré series

$$
P_{s}^{\prime \prime}(x K, y K)=\sum_{\gamma \in \Gamma} e^{-d_{s}^{\prime \prime}(x K, \gamma y K)} \quad \forall s>0, \forall x, y \in G
$$

and its critical exponent

$$
\delta^{\prime \prime}(\Gamma)=\inf \left\{s>0 \mid P_{s}^{\prime \prime}(x K, y K)<+\infty\right\} .
$$

It follows from (3.2) that

$$
0 \leq \delta(\Gamma) \leq \delta^{\prime \prime}(\Gamma) \leq \delta^{\prime}(\Gamma) \leq 2\|\rho\| .
$$

Proof of Theorem 1.4. In the proof of Theorem 1.3 and Corollary 2.10, we compared the series (2.20), or equivalently (2.22), with the Poincaré series (1.1) and (2.7). If we consider instead the Poincare series (3.3), we obtain in the same way that $(2.22)$ is bounded from above by $P_{\|\rho\|+\zeta}^{\prime \prime}(x K, y K)$ and from below by $P_{\|\rho\|+\zeta+\varepsilon}^{\prime \prime}(x K, y K)$, for every $\varepsilon>0$. Hence (2.22) converges if $\zeta>\delta^{\prime \prime}(\Gamma)-\|\rho\|$, while (2.22) diverges if $\zeta<\delta^{\prime \prime}(\Gamma)-\|\rho\|$. We conclude as in the abovementioned proof.

Acknowledgements. The authors are grateful to the referee for checking carefully the manuscript and making several helpful suggestions of improvement. The second author acknowledges financial support from the University of Orléans during his Ph.D. and from the Methusalem Programme Analysis and Partial Differential Equations during his postdoc stay at Ghent University.

\section{REFERENCES}

[Alb99] P. Albuquerque, Patterson-Sullivan theory in higher rank symmetric spaces, Geom. Funct. Anal. 9 (1999), 1-28, MR1675889.

[AAS10] B. Amri, J.-P. Anker, and M. Sifi, Three results in Dunkl analysis, Colloq. Math. 118 (2010), 299-312, MR2600531.

[AnJi99] J.-P. Anker and L. Ji, Heat kernel and Green function estimates on noncompact symmetric spaces, Geom. Funct. Anal. 9 (1999), 1035-1091, MR1736928.

[Ank90] J.-P. Anker, $\mathbf{L}_{p}$ Fourier multipliers on Riemannian symmetric spaces of the noncompact type, Ann. of Math. (2) 132 (1990), 597-628, MR1078270.

[Ank91] J.-P. Anker, The spherical Fourier transform of rapidly decreasing functions. A simple proof of a characterization due to Harish-Chandra, Helgason, Trombi, and Varadarajan, J. Funct. Anal. 96 (1991), 331-349, MR1101261.

[Ank92] J.-P. Anker, Sharp estimates for some functions of the Laplacian on noncompact symmetric spaces, Duke Math. J. 65 (1992), 257-297, MR1150587.

[AnOs03] J.-P. Anker and P. Ostellari, The heat kernel on noncompact symmetric spaces, in: Lie groups and symmetric spaces, vol.210, 27-46, Amer. Math. Soc. Transl. Ser. 2, Amer. Math. Soc., Providence, RI, 2003, MR2018351. 
[Cor90] K. Corlette, Hausdorff dimensions of limit sets. I, Invent. Math. 102 (1990), 521541, MR1074486.

[DaMa88] E. B. Davies and N. Mandouvalos, Heat kernel bounds on hyperbolic space and Kleinian groups, Proc. London Math. Soc. (3) 57 (1988), 182-208, MR940434.

[Els73a] J. Elstrodt, Die Resolvente zum Eigenwertproblem der automorphen Formen in der hyperbolischen Ebene. I, Math. Ann. 203 (1973), 295-300, MR360472.

[Els73b] J. Elstrodt, Die Resolvente zum Eigenwertproblem der automorphen Formen in der hyperbolischen Ebene. II, Math. Z. 132 (1973), 99-134, MR360473.

[Els74] J. Elstrodt, Die Resolvente zum Eigenwertproblem der automorphen Formen in der hyperbolischen Ebene. III, Math. Ann. 208 (1974), 99-132, MR360474.

[Hel00] S. Helgason, Groups and geometric analysis, Mathematical Surveys and Monographs, American Mathematical Society, Providence, RI, 2000, MR1790156.

[Kna02] A. W. Knapp, Lie groups beyond an introduction, Progress in Mathematics, Birkhäuser Boston, Inc., Boston, MA, 2002, MR1920389.

[Kni97] G. Knieper, On the asymptotic geometry of nonpositively curved manifolds, Geom. Funct. Anal. 7 (1997), 755-782, MR1465601.

[Leu03] E. Leuzinger, Kazhdan's property $(\mathrm{T}), L^{2}$-spectrum and isoperimetric inequalities for locally symmetric spaces, Comment. Math. Helv. 78 (2003), 116-133, MR1966754.

[Leu04] E. Leuzinger, Critical exponents of discrete groups and $L^{2}$-spectrum, Proc. Amer. Math. Soc. 132 (2004), 919-927, MR2019974.

[Pat76] S. J. Patterson, The limit set of a Fuchsian group, Acta Math. 136 (1976), 241-273, MR450547.

[Str81] J.-O. Strömberg, Weak type $L^{1}$ estimates for maximal functions on noncompact symmetric spaces, Ann. of Math. (2) 114 (1981), 115-126, MR625348.

[Sul87] D. Sullivan, Related aspects of positivity in Riemannian geometry, J. Differential Geom. 25 (1987), 327-351, MR882827.

[Web08] A. Weber, Heat kernel bounds, Poincaré series, and $L^{2}$ spectrum for locally symmetric spaces, Bull. Aust. Math. Soc. 78 (2008), 73-86, MR2458299.

JEAN-PhILIPPE ANKeR: anker@univ-orleans.fr

Institut Denis Poisson, Université d'Orléans, Université de Tours \& CNRS, Orléans, France

Hong-Wei ZHANG: hongwei.zhang@ugent.be

Department of Mathematics, Ghent University, Ghent, Belgium

Institut Denis Poisson, Université d'Orléans, Université de Tours \& CNRS, Orléans, France 